\title{
EL DICTAMEN DE CAPACIDADES \\ DENTRO DEL PROCEDIMIENTO DEL RECONOCIMIENTO LEGAL DE LA DISCAPACIDAD: LA EXPERIENCIA EN LA COMUNIDAD DE MADRID
}

\author{
Autores: Carlos de Fuentes García-Romero de Tejada \\ carlosdefuentes@pdi.ucm.es \\ Universidad Complutense \\ Juan Manuel Sevillano Martín \\ jmsevillano@icade.comillas.edu \\ Técnico Orientación Profesional en Centro Base de la Comunidad de Madrid
}

\section{Resumen}

La Ley General de derechos de las personas con discapacidad y de su inclusión social de 2013 en su artículo 12.3.a) trata por primera vez sobre la emisión de un posible dictamen de capacidades en el proceso de calificación y reconocimiento del grado de discapacidad. Los Centros Base de la Comunidad de Madrid llevan realizando este dictamen desde hace algunos años y no sólo se fijan en las limitaciones sino también recaban las capacidades y las fortalezas de las personas que 
atienden muy en la línea de la Convención de Naciones Unidas de Derechos de las Personas con Discapacidad de 2006. El presente artículo analiza esta experiencia para concluir que esta herramienta puede ser muy interesante para lograr una mejor orientación laboral de las personas con discapacidad y facilitar la accesibilidad y adaptación de puestos de trabajo y, por ello, debería generalizarse su uso.

Palabras clave: dictamen de capacidades; personas con discapacidad; reconocimiento legal de discapacidad; Centros Base de la Comunidad de Madrid; orientación laboral.

The capacity report within the procedure of the Legal Recognition of the Disability: the experience in the Community of Madrid

\begin{abstract}
The General Law on the Rights of Persons with Disabilities and their social inclusion of 2013 in its article 12.3.a) speaks for the first time of the capacity report in the process of recognition of the degree of disability. The Community of Madrid Base Centers has been carrying out this opinion for a few years and not only focuses on the limitations and also collects the capacities and strengths of the people who serve, in line with the United Nations Convention on the Rights of Persons with Disabilities in 2006. This article analyzes this experience to conclude that this tool can be very interesting to achieve a better job orientation of people with disabilities and facilitate accessibility and adaptation of jobs and, therefore, should be generalized its use.
\end{abstract}

Key words: capacity report; people with disabilities; legal recognition of disability; Community of Madrid Base Centers; career guidance. 


\section{INTRODUCCIÓN}

La Ley General de derechos de las personas con discapacidad y de su inclusión social (en adelante, LGD) ${ }^{1}$, en su artículo 12.3.a) aborda por primera vez el tema de la emisión de un posible dictamen de capacidades en el proceso de calificación y reconocimiento del grado de discapacidad. En concreto, indica que le corresponde a los equipos multiprofesionales de calificación y reconocimiento del grado de discapacidad emitir un dictamen técnico sobre las deficiencias, las limitaciones para realizar las actividades y las barreras para la participación social, en el que se recoja, además, "las capacidades y habilidades para las que la persona necesita apoyos".

Este último inciso de este apartado del artículo 12 LGD se trata de una novedad que incorpora el Texto Refundido de la Ley General de discapacidad que puede tener cierta virtualidad jurídica, motivo por el cual nos vamos a detener a su análisis teórico-práctico en este artículo, partiendo de la experiencia en los Centros Base de la Comunidad de Madrid.

Los Centros Base de Atención a personas con discapacidad llevan a cabo la valoración y orientación al colectivo otorgando el Reconocimiento del Grado de discapacidad en la Comunidad de Madrid; pretenden informar y facilitar a cada persona el camino más rápido y seguro para su inclusión y mantenimiento de un puesto de trabajo. Para realizar esta tarea, el personal del Área de Orientación Profesional (Técnico/a en Orientación Laboral y Técnico/a en Pedagogía) aplica la metodología VOIL (Valoración, Orientación e Inserción Laboral) ${ }^{2}$ que se fundamenta en la adecuación entre las capacidades de cada persona y las exigencias de la actividad laboral que quiere desempeñar. Con esta herramienta especializada se ofrece a las personas, sus familiares, entidades del sector de la discapacidad y empresas el asesoramiento necesario e información sobre procesos de selección y recursos de apoyo al empleo.

Por tanto, en el reconocimiento legal de la discapacidad los equipos de los Centros Base de la Comunidad de Madrid no sólo se fijan en las limitaciones de las personas con discapacidad (en adelante, PCD) sino también recaban las capacidades y las fortalezas de las personas que atienden. Analizaremos esta experiencia que está acorde con la nueva redacción del art. 12.3.a) LGD y estudiaremos su impacto y balance de actividad.

\footnotetext{
${ }^{1}$ Real Decreto Legislativo 1/2013, de 29 de noviembre, BOE de 3 de diciembre de 2013.

${ }^{2}$ Toda la información sobre esta metodología está accesible en: http://www.madrid.org/cs/Satellite $? c=C M \_I n f P r a c t i c a \_F A \& c i d=1142310100115 \&$ language $=$ es\&pagename $=$ ComunidadMadrid\%2FEs tructura\&pv $=1354190551426$
} 


\section{LA REFUNDICIÓN LLEVADA A CABO POR LEY GENERAL DE DISCAPACIDAD DE 2013}

Como es sabido, la Ley General de discapacidad se trata de un texto normativo que refunde tres leyes emblemáticas para las PCD como son, en primer lugar, la famosa LISMI, Ley 13/1982, de 7 de abril ${ }^{3}$, de integración social de las personas con discapacidad; en segundo término, la ley conocida con su acrónimo LIONDAU, Ley 51/2003, de 2 de diciembre ${ }^{4}$, de igualdad de oportunidades, no discriminación y accesibilidad universal de las PCD; y, por último, la Ley 49/2007, de 26 de diciembre ${ }^{5}$, en la que se establece el régimen de infracciones y sanciones en materia de igualdad de oportunidades, no discriminación y accesibilidad universal de las PCD. En esta labor refundidora, la LGD además ha armonizado y aclarado el contenido de la legislación de discapacidad teniendo como referente la Convención de Naciones Unidas sobre los Derechos de las Personas con Discapacidad ${ }^{6}$ (en adelante, la Convención) ${ }^{7}$.

El contenido del artículo 12.3.a) de la LGD que se estudia viene a refundir el artículo 10 de la LISMI en el cual no viene recogido el inciso antes expuesto en el que se habla sobre la posibilidad de emitir un dictamen de capacidades en el momento del proceso de calificación del grado de discapacidad. Es, por tanto, un inciso nuevo en la legislación española pero que pasó inadvertido a los ojos del Consejo Económico y Social en su dictamen previo sobre esta norma (Consejo Económico y Social, 2013, p. 8). Entendemos que habría que verlo como un añadido necesario para adecuar nuestra legislación a la Convención Internacional de la $\mathrm{ONU}^{8}$. En efecto, la LGD promueve que en el momento del reconocimiento legal de la discapacidad no sólo haya que fijarse en las barreras sociales y en las limitaciones que la PCD presenta por la interacción con dichos obstáculos, sino que los equipos multiprofesionales deban completar su dictamen de calificación de la discapacidad con las capacidades y habilidades que aún atesora la $\mathrm{PCD}$, pese a las limitaciones que le provocan la discapacidad, incluso cuando éstas - las habilidades y capacidades- puedan precisar de algún apoyo. De esta manera entendemos nosotros la redacción un tanto confusa del precepto analizado.

\footnotetext{
${ }^{3}$ Publicada en BOE de 30 de abril de 1982.

${ }^{4}$ Publicada en BOE de 3 de diciembre de 2003.

${ }^{5}$ Publicada en BOE de 27 de diciembre de 2007.

${ }^{6}$ Aprobada por la Asamblea de la ONU el 13 de diciembre de 2006, junto con su Protocolo Facultativo, ratificada por España mediante instrumento de ratificación publicado en el BOE de 21 de abril de 2008.

${ }^{7}$ Vid. párrafos undécimo y duodécimo de la Exposición de motivos de la LGD.

${ }^{8}$ En el mismo sentido, pero sin desarrollar la idea, De Lorenzo García (2015, p. 59).
} 


\section{LA CONEXIÓN CON LA CONVENCIÓN DE LA ONU}

Como se acaba de indicar, la nueva función que el art. 12.3.a) de la LGD encomienda a los equipos de valoración de la discapacidad para que tengan presente las capacidades y habilidades que la PCD puedan albergar entendemos que conecta nuestra legislación con la Convención de la $\mathrm{ONU}$ y ello porque es una muestra de concebir a las PCD como sujetos titulares de derechos que pueden ejercer de acuerdo con el principio de la libertad con la toma de decisiones (exposición de motivos de la LGD, en consonancia con la letra 'n' del preámbulo de la Convención). El hecho de tomar en consideración en el momento de la emisión del reconocimiento legal de la discapacidad no sólo las limitaciones y carencias sino lo que son capaces y sus potencialidades, a lo que nosotros añadiríamos identificar las necesidades de apoyo que precisan las PCD para sortear las barreras sociales que les impiden tener una inclusión social equiparable con el resto de la sociedad ${ }^{9}$, denota un cambio muy trascedente de perspectiva. En definitiva, capacidades, habilidades y apoyos, tanto los que cuentan ya las PCD como aquéllos que puedan precisar, deben ser relacionados en un dictamen por los equipos de valoración. Ello está en mayor consonancia con la Convención que un reconocimiento legal de discapacidad que no los tenga en cuenta. Entre otras, esta concepción se ve incardinada con las letras 'e' (la discapacidad evoluciona), 'm' (poner en valor las contribuciones que pueden realizar las PCD), 'n' (libertad en la toma de decisión, antes ya referida) y 'o' (oportunidad de participar activamente) del preámbulo de la Convención y con sus artículos 1 (promover, proteger y asegurar el goce pleno de los derechos humanos y libertades fundamentales), 3 'c' (principio general de la participación e inclusión plenas), 12.2 (capacidad jurídica en igualdad de condiciones con los demás) y 27 (derecho al trabajo y empleo).

En definitiva, las PCD aportan a la sociedad y lo hacen con las cualidades, facultades y competencias que pueden atesorar. Es momento de fijarse más en ellas y en los apoyos que precisan, en lugar de poner el foco de atención en las limitaciones y lagunas que provoca la discapacidad. Y hay que hacerlo desde el momento del reconocimiento legal de la discapacidad. Tal y como ocurre en el Derecho de la Dependencia, de nada sirve que se reconozca una discapacidad si a la vez no se valoran las capacidades, se tienen en cuentan los apoyos que la persona precisa y se informa de los servicios que se ponen a su disposición para promover, proteger y asegurar el goce pleno de su participación e inclusión plenas.

\footnotetext{
${ }^{9}$ En relación a los apoyos para la PCD, vid. De Fuentes $\mathrm{G}^{\mathrm{a}}$-Romero de Tejada (2016).
} 


\section{3. ¿POR QUÉ, PARA QUÉ Y CUÁNDO UTILIZAR EL DICTAMEN DE CAPACIDADES?}

Identificada la nueva competencia que la LGD encomienda a los equipos de valoración de la discapacidad, es momento de preguntarse la virtualidad que esta misión conlleva frente al actual reconocimiento legal de la discapacidad, qué sentido puede tener, cuándo utilizarlo y con qué finalidad.

Actualmente, la valoración y calificación de la discapacidad se ejecuta utilizando como norma básica el Real Decreto 1971/199910 según la cual (por ejemplo, Anexo $1 \mathrm{~A}$, capítulo $1,4^{\circ}$ ), las pautas de valoración se fundamentan en el efecto que la deficiencia haya tenido en la capacidad de la persona para poder llevar a cabo las actividades de la vida diaria, es decir, en el grado de discapacidad que ha originado tal deficiencia. Y, por tanto, se pone el foco de atención en la limitación de la capacidad más que en reconocer las capacidades residuales, sus habilidades y las necesidades de apoyo que la persona precisa para superar las barreras sociales con las que la persona interactúa.

En un enfoque de derechos humanos de la discapacidad, tal como indica Asís, la protección jurídica de la misma debe tender a la consecución de los planes de vida de las PCD, que en definitiva expresa el logro de una vida humana digna y el resultado de la elección personal de cómo se quiere vivir. No obstante, en la actualidad se sigue considerando a la PCD como una persona menos válida ${ }^{11}$ de ahí, entendemos nosotros, la importancia de que el Derecho de la Discapacidad se asiente en mayor medida en tomar en consideración la capacidad, necesidades de apoyos y potencial de las PCD tal y como se está haciendo en los Centros Base de la Comunidad y que pasamos a analizar a continuación.

Para concluir con esta parte introductoria hemos de indicar que entendemos que el dictamen de capacidades debe utilizarse al menos y fundamentalmente:

1) para situaciones de discapacidad sobrevenida para las cuales puede ayudar a asumir mejor su nueva condición de PCD;

y 2) para PCD en edad laboral, en las que puede servir, por un lado, para un mejor ajuste de la persona con las ocupaciones que pretende ocupar y, por otro, para aclarar situaciones de compatibilidad entre pensiones y trabajo. En efecto, en estos momentos ocurren casos en los que una PCD a la que se le ha declarado una Incapacidad Permanente Absoluta pretende volver al trabajo y la situación de inseguridad jurídica es evidente; son múltiples

\footnotetext{
${ }^{10}$ Real Decreto 1971/1999, de 23 de diciembre, de procedimiento para el reconocimiento, declaración y calificación del grado de minusvalía, BOE de 26 de enero de 2000.

${ }^{11}$ Vid. los diversos trabajos del autor que tratan sobre el particular, entre otros, De Asís (2015, pp. 75-84).
} 
los casos en los que el trabajador es privado de la pensión que percibía por realizar una nueva actividad laboral y ello promueve el inmovilismo laboral de estas PCD pese a la posibilidad de volver a recuperar su capacidad productiva y un puesto de trabajo ${ }^{12}$. Es indudable que el Equipo de Valoración de Incapacidades de la Seguridad Social podría utilizar el dictamen de capacidades emitido por el organismo competente en la materia, el Centro Base de la Comunidad Autónoma que corresponda, para ayudarle a estudiar y valorar la situación del trabajador con discapacidad.

\section{LA PRÁCTICA DEL DICTAMEN DE CAPACIDADES EN LA COMUNIDAD DE MADRID}

\section{ANTECEDENTES}

El dictamen de capacidades proviene de una longeva colaboración entre el IMSERSO (organismo del Ministerio de Sanidad y Servicios Sociales) y la Consejería de Políticas Sociales y Familia de la Comunidad de Madrid. Parte de una metodología de trabajo desarrollada en la década de los 90 del siglo pasado que avanzó un programa informático denominado ESTRELLA ${ }^{13}$ y que pretendía objetivar las capacidades y competencias de las PCD, de forma que acercase el concepto de "ocupaciones ajustadas" tanto a los profesionales de la Orientación de los Centros Base, como a ofrecer a las personas con discapacidad alternativas de inserción laboral acordes a las posibilidades de la persona.

Posteriormente, el año 2003, año europeo de las personas con discapacidad, significó un punto de inflexión en la incorporación de paradigmas de integración y posteriormente de inclusión laboral de las PCD. En aquella época aún se cuestionaban las posibilidades de inserción laboral de la mayoría del colectivo pues aunque la LISMI había avanzado hacia el concepto de integración, aún no se había generalizado en la sociedad en general.

El año Europeo sí supuso un verdadero impulso, de forma que surgieron Planes de Acción para Personas con discapacidad ${ }^{14}$ que buscaban la operatividad

\footnotetext{
${ }^{12}$ Vid. sobre el particular, entre otros, Sempere Navarro (2013) y De Fuentes G⿳a-2 -Romero de Tejada (2016).

${ }^{13}$ Sobre el marco teórico de este programa informático vid. IMSERSO-COCEMFE-COMUNIDAD DE MADRID, mayo de 2006, accesible en: http://www.imserso.es/InterPresent2/groups/imserso/documents/ binario/marco_teorico.pdf

${ }^{14}$ En la Comunidad de Madrid, aunque se gestó años antes se puso en marcha en el Plan 2005. 2008, accesible en: http://www.madrid.org/bvirtual/BVCM007014.pdf. A nivel nacional, se implementó el II Plan de Acción para PCD 2003-2007, toda la información accesible en: http://sid.usal.es/idocs/F8/ FDO6636/iipapcd2003_2007.pdf
} 
no solo hacia la obtención de un empleo, sino que la búsqueda fuera definida, ajustada y concreta: todo ello para poder aumentar las garantías del éxito en la inserción laboral del colectivo.

El programa informático ESTRELLA evolucionó hacia la aplicación informática denominada Valoración, Orientación e Integración Laboral de Personas con discapacidad, conocida con el acrónimo VOIL. Esta aplicación definió quince factores de capacidad con criterios de la Clasificación Internacional de Funcionamiento de la Discapacidad y la Salud de la OMS, en adelante CIF. Se puede decir que los factores de capacidad fueron por delante de la Convención de Derechos de la ONU y tanto más de nuestra vigente LGD de 2013.

Dicha aplicación informática VOIL establece a través de estos factores de capacidad, así como de los posibles agentes de riesgo, un método o manera que puede mejorar la obtención de un puesto de trabajo ajustado, lo que ofrece una mayor garantía de éxito a la PCD. Se puede decir que esta es la gran aportación que ofrece el Dictamen o Perfil de Capacidades y la aplicación VOIL.

El acercamiento a esta metodología además facilita la ruptura de estereotipos y estigmas infundados, pero muy generalizados, de manera que muestra las capacidades y las posibilidades de inserción de todas y cada una de las PCD, independiente de si su discapacidad se debe a una limitación física, sensorial, intelectual o por salud mental. Se remarca el significado de "todas" las personas puesto que el conjunto del colectivo podría potencialmente desarrollar una actividad laboral (no significa a jornada completa o un empleo con funciones complejas); esta aplicación muestra de forma más objetiva las posibilidades de desempeño, así como las posibles adaptaciones que se podrían ofrecer.

Por una parte, el dictamen de capacidades, permite obtener ocupaciones que objetivamente no ven comprometido su desempeño observando las limitaciones de la persona; por otra parte, si transformamos el dictamen de capacidades en "Requerimientos de Puesto" permite objetivar funciones de los puestos de trabajo para de esta forma poder acercarnos al tan necesario paradigma del Ajuste "Persona-Puesto". Los mismos factores de capacidad que definen el dictamen de capacidades son los que definen los Requerimientos del Puesto, de forma que la propia aplicación nos facilita de forma automática si pude existir un "conflicto" leve, moderado o grave en el desempeño del puesto de trabajo; de manera que nos focaliza para poder definir si procede adaptación, o realmente la limitación compromete el desempeño del puesto de trabajo.

Actualmente, en éste nuestro siglo XXI, se considera superado el paradigma de buscar los mejores candidatos basándonos únicamente en las competencias formales (cualificación y/o experiencia), dejando fuera habilidades, capacidades 
y aptitudes ${ }^{15}$. Si cada vez más la gestión de Recursos Humanos busca conocer habilidades personales, desempeño, intereses, es decir, la felicidad y satisfacción que produce la realización a través del empleo, la persona seleccionada no sólo tendrá que poseer las aptitudes para el desempeño, sino también las actitudes y el deseo de llevarlas a cabo. No hace falta poner ejemplos de desempeño en personas empleadas en puestos que no desean, para poder dejar claro de lo que estamos hablando. Sin embargo, sí pueden ser esclarecedores ejemplos de PCD en empleos que sirven para la absoluta realización de los mismos, lo que hace que los lleven a cabo felices y con la satisfacción que produce la realización profesional.

\section{DESCRIPCIÓN DE LOS FACTORES DE CAPACIDAD}

Los quince factores de capacidad que el Equipo de Valoración y Orientación del Centro Base puede tener en cuenta son los siguientes:

$\checkmark$ Destreza manual: Capacidad para realizar actividades que requieran acciones coordinadas, con precisión y rapidez manual. Correspondencias con la CIF: $d 440$ Uso fino de la mano, d445 Uso fino de la mano y el brazo.

$\checkmark$ Desplazamiento: Capacidad para andar, caminar, pasear, moverse y/o desplazarse, utilizando o no algún tipo de equipamiento. Incluye: Recorridos cortos y largos, subir y bajar escaleras, correr, trepar, saltar. Correspondencias con la CIF: $d 450$ hasta $d 475$.

$\checkmark$ Acceso al transporte: Capacidad para acceder (acceso físico) a transporte público o hacer uso de vehículo particular. Correspondencias con la CIF: d470 hasta 475.

$\checkmark$ Posición: Capacidad para adoptar y mantener una postura determinada. Correspondencias con la CIF: d410 hasta $d 420$.

$\checkmark$ Fuerza: Capacidad para realizar esfuerzos físicos (ejercicio físico / manipulación de pesos y/o de objetos de gran volumen). Correspondencias con la CIF: $d 430$ hasta $d 435, d 445$.

$\checkmark$ Tolerancia: Capacidad, a efectos de este método de valoración, para soportar situaciones de estrés tensión y/o fatiga mental, provocadas por la realización de tareas monótonas, rutinas laborales, exigencia de alta productividad, etc., que requieran concentración y/o atención continuada. Correspondencias con la CIF: d240.

${ }^{15}$ Sobre el tema y los problemas deontológicos de administrar test de personalidad, vid. COLEGIO OFICIAL DE PSICÓLOGOS DE MADRID, Guía Técnica y de Buenas Prácticas en Reclutamiento y Selección de Personal, accesible en: http://www.copmadrid.org/webcopm/recursos/guiatecnicabuenaspracticas.pdf 
$\checkmark$ Autonomía personal: Capacidad para actuar con independencia no necesitando supervisión en la realización de actividades relacionadas con el autocuidado y la percepción de riesgos. Correspondencias con la CIF: d510 hasta d570, d620 hasta d640.

$\checkmark$ Relaciones interpersonales: Capacidad básica para iniciar y mantener relaciones con otras personas, a efectos de participar en un grupo relacionado con la formación y/o el empleo. (Si la opción seleccionada es Dificultad Grave / Completa, la Aplicación automáticamente no dará opción a su inclusión Bolsa de Empleo/Trabajo). Correspondencias con la CIF: d710.

$\checkmark$ Orientación en el entorno: Se refiere a la capacidad para orientarse en el medio donde vive (barrio, ciudad...) y utilizar (conocer y usar) el transporte público existente (metro, autobús, etc.). Correspondencias con la CIF : d460, d470.

$\checkmark$ Manejo de dinero: Capacidad para participar en transacciones económicas básicas, cambio de moneda, realización de pagos y devoluciones a otras personas. Correspondencia con la CIF : $d 860$.

$\checkmark$ Aprendizaje: Capacidad de adquirir conocimientos y/o competencias, a través del estudio y/o la práctica (centrando la atención, encontrando soluciones a problemas, eligiendo una opción entre varias...) para la realización de una actividad o conjunto de actividades nuevas. Correspondencias con la CIF : d130 hasta d155, d160 hasta d177.

$\checkmark$ Visión: Capacidad visual, utilizando las ayudas técnicas de que disponga, (leer periódicos, libros, pantallas de ordenador, carteles en la calle, en el interior, en el transporte público, de noche o en lugares con poca iluminación...), así como reconocer personas y discriminar objetos, detalles y/o colores. Incluye fundamentalmente la capacidad que tiene la persona para ver, a la hora de realizar actividades relacionadas con la vida diaria, la formación y/o el empleo. (Excluye: en este factor no se valoran otras capacidades con las que la persona pueda suplir las limitaciones que tenga en relación con la visión.). Correspondencia con la CIF: d110.

$\checkmark$ Audición: Capacidad para oír, reconocer y discriminar sonidos, utilizando las prótesis auditivas de que disponga. Incluye fundamentalmente la capacidad para oír (sonidos y/o a otras personas), a la hora de realizar actividades relacionadas con la vida diaria, la formación y/o el empleo. Excluye: en este factor no se valoran la Comprensión Verbal y/u otras capacidades con las que la persona pueda suplir las limitaciones que tenga en relación con la audición. Correspondencia con la CIF: d115.

$\checkmark$ Comprensión: Capacidad para entender mensajes orales (excluye audición). Correspondencias con la CIF: d310 hasta d325, d350 hasta d360.

$\checkmark$ Expresión oral: Capacidad para expresar mensajes orales. Correspondencias con la CIF: d330 hasta d345, d350 hasta d360. 
Cada uno de estos factores se ajustan en tres posibles grados de limitación:

$\checkmark$ 10. No existe dificultad

$\checkmark$ 20. Dificultad Ligera / Moderada.

$\checkmark$ 30. Dificultad Grave / Completa.

Cabe recordar que la discapacidad o diversidad funcional de cada persona o de cada limitación es muy difícil de ajustar, de manera que esta metodología puede aportar luz hacia la idea de poder objetivar posibles "ajustes razonables" en el puesto de trabajo; o bien definir si la dificultad de los ajustes puede llevar hacia el no desempeño de una determinada ocupación laboral.

Por otra parte, si entendemos a la persona como un todo, nos permite salir de las tan empleadas etiquetas genéricas que hablan de la discapacidad física, psíquica (intelectual y salud mental) y sensorial. Es fundamental romper estos estereotipos, ya que las personas en la mayoría de los casos no tenemos sólo una limitación, de forma que ya estaríamos siendo inexactos en la etiqueta, sino porque además, perpetúan equívocos relativos especialmente a limitaciones debidas a salud mental y a la discapacidad intelectual.

Si dejamos de hablar de este tipo de etiquetas estaríamos hablando de personas con diversidad funcional y diferentes niveles de apoyo (De Fuentes $\mathrm{G}^{\mathrm{a}}$-Romero de Tejada, 2016) a las que el dictamen de capacidades facilita no sólo la integración, o mejor aún inclusión laboral, sino también aporta luz en los casos de Reorientación y Recualificación Profesional, especialmente en los casos de Incapacidades Permanentes de Seguridad Social.

Podría ser objeto de estudio, o mejor dicho, sería necesario implementar éste u otro tipo de Reorientación Profesional que ofrezca luz a las personas a las que Seguridad Social les otorga la condición de Pensionistas de Incapacidad Permanente en ocasiones aún con largas carreras laborales de cotización por delante. En la mayoría de estos casos, las personas se encuentran con una limitación sobrevenida que las incapacita al menos para el desempeño de la profesión que venían desempeñando, lo que la Seguridad Social considera su "profesión habitual". Muchos casos con aún una larga vida laboral por delante, y sin embargo, con una edad avanzada para, de manera sorpresiva y no planificada, comenzar a recualificarse profesionalmente. Desde la experiencia de los recursos especializados (Centros Base, Centros de Rehabilitación Laboral de atención a personas con problemas de salud mental grave, Centros Ocupacionales para PCD intelectual, etc.) se observa que no sólo es necesario generar una cobertura económica (pensión), también es absolutamente necesario reorientar a estas personas, que se encontraban desempeñando su profesión y las cuales se ven privadas de dicho quehacer y del potencial de realización personal y profesional que el trabajo confiere, estando en muchas ocasiones en plena madurez laboral. 
En cuanto a los criterios para definir el grado de limitación o las posibles adaptaciones a plantear, cabe destacar que los Equipos de Valoración y Orientación únicamente tendrán en cuenta los factores en los que los profesionales tengan conocimiento de limitación, de manera que la norma general en el resto de factores, será la que se presupone a cualquier persona sin discapacidad.

\section{CONCLUSIONES}

En los casi quince años en los que se ha trabajado en los Centros Base de Atención a Personas con Discapacidad de la Comunidad de Madrid, se puede decir que se ha avanzado hacia el paradigma del Ajuste "Persona-Puesto" mejorando en la resolución de situaciones de Aptitud/No Aptitud y ofreciendo una respuesta uniforme y con criterios objetivos en función del dictamen de capacidades enfrentado al Perfil de Requerimientos del Puesto de Trabajo.

Si aceptamos el dictamen de capacidades como la "moneda de curso legal" que define, concreta, ajusta y, en definitiva, mejora la orientación, estaremos apostando por un sistema que ofrece ocupaciones y propuestas formativas ajustadas, facilitando la accesibilidad y que permite avanzar, incluso, hacia los ajustes razonables. Al objetivar Capacidades de la persona frente a Requerimientos del puesto (medidos igualmente en estos mismos factores de capacidad) estamos aceptando que la inclusión laboral de todas las PCD es posible. Esto nos lo confirma la propia evolución vivida desde la promulgación de la LISMI en el año 1982, hasta la actualidad. Es importante remarcar que ya nadie se plantea hoy que las personas con discapacidad puedan tener un espacio de desempeño laboral más o menos adaptado.

Como posibilidades de mejora planteamos que se abra esta "Moneda de curso legal", de manera que pueda ser conocida y usada por profesionales que muchas veces son los que mejor conocen a las PCD. Este Perfil o dictamen de capacidades podrá ser realizado, por ejemplo, por los Profesionales de Centros Ocupacionales, Centros de Rehabilitación Laboral e incluso por profesionales de Centros de Rehabilitación Psicosocial y/o Centros de Día. Asimismo, sería idóneo que el dictamen de capacidades fuese utilizado por todos los servicios públicos de empleo que puedan apoyar a las PCD en su camino a la obtención de un trabajo ${ }^{16}$. Todo ello para poder realizar una Orientación, Reorientación, Ajuste, Reajuste o Recualificación que partiendo siempre de los intereses de la persona le facilite un

\footnotetext{
${ }^{16}$ En este sentido, indicar que hubo una experiencia piloto en la Comunidad de Madrid de coordinación y uso de la herramienta informática VOIL entre el Servicio Regional de Empleo y los Centros Base. Sería idóneo poder retomar y extender esta práctica de colaboración entre organismos de apoyo al empleo.
} 
Itinerario Formativo Laboral (Ceniceros Estévez \& Oteo Antuñano, 2003) que logre hacer realidad el principio de la igualdad de oportunidades y de resultado ${ }^{17}$ que establecen tanto la Convención de la ONU de 2006 como nuestra LGD, marco legal de referencia de obligado cumplimiento.

Que el empleo ha cambiado en los últimos años, es algo con lo que estamos todos de acuerdo ${ }^{18}$. Han cambiado las formas de empleo (teletrabajo, empleos deslocalizados, uso masivo de tecnologías de la información y las comunicaciones, formas de control empresarial, etc.), y también se han transformado hasta las propias funciones de los grupos profesionales de los convenios colectivos.

El teletrabajo, hoy en día, se entiende de forma diferente a como se definió hace quince años, y además avanza a pasos agigantados, incorporando variables de sostenibilidad (menores desplazamientos en ciudades contaminadas), corresponsabilidad familiar (mal llamada conciliación) y sobre todo ofreciendo la posibilidad de humanizar el trabajo. La deslocalización, la globalización cada día se entiende más como una oportunidad, de manera que se puede diseñar un coche en Japón o Alemania, se puede manufacturar en China, distribuir por todo el mundo en menos de un mes y acabar siendo facturado en Irlanda o Panamá... Si abrimos la mente hacia esta concepción del trabajo debemos apostar por un mundo laboral verdaderamente accesible en el que TODAS las personas, como antes ya dijimos remarcando el adjetivo del conjunto completo del colectivo, sean susceptibles de poder desarrollar una actividad laboral (repetimos que no nos referimos que todo el mundo lo haga a jornada completa o desempeñando un empleo con funciones complejas), en un ejemplo de la mejor Responsabilidad Social de las empresas ${ }^{19}$.

Este siglo nos ha digitalizado, automatizado, robotizado, hasta el punto de estar atisbando las relaciones humanas de carácter virtual. Todo ello modifica las relaciones laborales que se han flexibilizado en gran medida ${ }^{20}$. Nos permite ajustar en unos casos los puestos de trabajo teniendo en cuenta las graves limitaciones de la persona (situaciones con mayor dificultad de inserción laboral), o en otros casos objetivar limitaciones de la persona para poder adaptar el puesto de trabajo, que es lo que sucede en la mayoría de casos. Esta última forma es actualmente mucho más posible, en la mayoría de los casos con creatividad y las oportunidades que aporta la digitalización, automatización, virtualización, mecanización...en definitiva, la tecnología.

\footnotetext{
${ }^{17}$ Extensamente sobre la igualdad material y derecho a la no discriminación de las PCD y los diferentes niveles existentes, en De Fuentes $\mathrm{G}^{\mathrm{a}}$-Romero de Tejada (2016, pp. 61-90).

${ }^{18}$ Sobre el tema, vaticinando los cambios que luego han acaecido, vid., entre otros, Reich (1993, pp. $21,75,81,82,101-104)$ y Casas Baamonde et al. (1999).

${ }^{19}$ Sobre la Responsabilidad Social, vid. el importante trabajo de Sánchez-Urán \& Grau Ruiz (2013).

${ }^{20}$ Recuérdese el concepto de flexiguridad o flexiseguridad, preconizado por la Unión Europea, es la base de la reforma laboral de 2012 a cuya exposición de motivos nos remitimos.
} 
El Informe de la UNESCO de la Comisión Internacional sobre la Educación para el Siglo XXI, que presidió Jaques Delors a finales de los años 90 del siglo pasado (Delors, 1996) ya planteaba como nuevo paradigma la "Formación a lo largo de la vida del ser humano". Con la perspectiva que tenemos hoy, acertó completamente. El ser humano nunca antes había adquirido tanta cualificación y formación, y nunca antes ésos aprendizajes habían quedado obsoletos en tan poco tiempo. Esto ahonda en la idea de la rápida evolución en la que vivimos actualmente y ofrece tantas posibilidades que la funcionalidad en el empleo quedaría definida por la rápida evolución de las propias funcionalidades en los puestos de trabajo, e igualmente las adaptaciones que hace pocos años se planteaban como imposibles o complicadas, pueden llevarse a cabo con pequeñas modificaciones. Si ponemos a un periodista de 1980 a ejercer su profesión en la actualidad... igual no comprendería muy bien su función como gestor de redes sociales o como gestor de noticias que en menos de cinco minutos han podido dar la vuelta al mundo.

El dictamen de capacidades ayuda a objetivar mejor qué potencialidades atesoran las PCD para poder realizar una determinada actividad profesional y puede adaptarse con el paso del tiempo al ser un dictamen con vocación dinámica.

Creemos oportuno dejar una pregunta en el aire: en este momento en el que parece que peligra el sistema de Seguridad Social y/o de pensiones, ipodemos permitirnos dejar fuera del mundo laboral a un colectivo que, de media, tiene tasas de actividad más de cuarenta puntos inferior al de las personas sin discapacidad y cuya tasa de desempleo está en torno al 28,6\%, muy superior al resto de la población? ${ }^{21}$ Si estas personas cotizasen al sistema de Seguridad Social seguro que sería muy interesante económicamente hablando.

El futuro es prometedor, aunque no se va a cambiar si no comenzamos cada una y cada uno de nosotros en nuestro día a día. Los medios están, las aplicaciones también, las facilidades tecnológicas nos están esperando. ¿Entonces? Igual lo que cuesta cambiar es nuestra forma de pensar, o lo que cada vez más se denomina "deconstruir" las estructuras mentales que no nos permiten afrontar y remover los obstáculos existentes para la inclusión laboral del colectivo de PCD. El dictamen de capacidades está definido y la experiencia de los Centros Base de la Comunidad de Madrid es una garantía. Es momento ahora de extender su uso para que logremos entre todos y todas poder dejar una sociedad más inclusiva para las generaciones futuras.

${ }^{21}$ Sobre las estadísticas del colectivo, vid. la serie anual Instituto Nacional de Estadística, El empleo de las personas con discapacidad, que se publica en diciembre de cada año con los datos relativos al año anterior, accesible en: http://www.ine.es 


\section{BIBLIOGRAFÍA}

\section{OBRAS DOCTRINALES}

Casas Baamonde, M. E., et al. (1999). Trabajo y empleo. Transformaciones del trabajo y futuro del Derecho del Trabajo en Europa. Valencia: Tirant lo Blanch.

Ceniceros Estévez, J. C. \& Oteo Antuñano, E. (2003). Orientación sociolaboral basada en itinerarios. Una propuesta metodológica para la intervención con personas en riesgo de exclusión. Madrid: Fundación Tomillo.

CIF (2001). Clasificación Internacional de Funcionamiento Discapacidad y Salud. Bilbao: Ministerio de Trabajo y Asuntos Sociales. Disponible en: http://www.imserso.es/InterPresent2/groups/imserso/documents/binario/435cif.pdf

Colegio Oficial de Psicólogos de Madrid (2011). Guía Técnica y de Buenas Prácticas en Reclutamiento y Selección de Personal. Madrid: Colegio Oficial de Psicólogos. Disponible en: http://www.copmadrid.org/webcopm/recursos/guiatecnicabuenaspracticas.pdf

Consejo Económico y Social (2013). Dictamen 9 "Sobre el Proyecto de Real Decreto legislativo por el que se aprueba el texto refundido de la Ley general de Derechos de las personas con discapacidad y de su inclusión social". Disponible en: http://www.ces.es/documents/10180/631510/Dic092013.pdf

De Asís, R. (2015). El encaje de la Convención Internacional de Derechos de las Personas con Discapacidad en el Texto Refundido. En F. Arenas Escribano \& M. A. Cabra de Luna (coords.), Comentarios al Texto Refundido de la Ley General de derechos de las personas con discapacidad y de su inclusión social (pp. 75-84). Madrid: La Ley.

De Fuentes $\mathrm{G}^{\mathrm{a}}$-Romero de Tejada, C. (2016). Compatibilidad de las prestaciones sociales por discapacidad y el empleo. Un medio para la inserción laboral de las personas con enfermedad mental grave. Cizur Menor: Thomson Reuters.

De Fuentes $\mathrm{G}^{\mathrm{a}}$-Romero de Tejada, C. (2016). Sobre el concepto jurídico de persona con discapacidad y la noción de apoyos necesarios. Revista Española de Discapacidad, 4(2), 81-99.

De Lorenzo García, R. (2015). Concepto y alcance de la refundición de textos legislativos. El mandato de refundición en el caso del Real Decreto Legislativo 1/2013, de 29 de noviembre. En F. Arenas Escribano \& M. A. Cabra de Luna, (coords.), Comentarios al Texto Refundido de la Ley General de derechos de las personas con discapacidad y de su inclusión social. Madrid: La Ley.

Delors, J. (1996). La educación encierra un tesoro. Informe a la UNESCO de la Comisión internacional sobre la educación para el siglo XXI. Madrid: Santillana-Ediciones Unesco. Disponible en: http://www.unesco.org/education/pdf/DELORS_S.PDF

IMSERSO-COCEMFE-Comunidad de Madrid (mayo, 2006). Marco teórico "Método Estrella". Disponible en: http://www.imserso.es/InterPresent2/groups/imserso/documents/binario/marco_teorico.pdf 
Instituto Nacional de Estadística. El empleo de las personas con discapacidad, que se publica en diciembre de cada año con los datos relativos al año anterior. Disponible en: http://www.ine.es

Manual Voil (2017). Manual Valoración, Integración y Orientación de Personas con discapacidad. Madrid: Comunidad de Madrid. Disponible en: http://www.madrid.org/bvirtual/BVCM014067.pdf

Reich, R. (1993). El trabajo de las naciones, (traducción Federico Villegas). Barcelona: Ed. Javier Vergara S.A.

Sánchez-Urán, Y. (dir.) \& Grau Ruiz, M. A. (coord.). (2013). Responsabilidad Social de las Organizaciones. Madrid: Editorial Grupo 5.

Sempere Navarro, A. V. (2013). El debate sobre incompatibilidad entre pensiones y trabajo productivo. Revista Aranzadi Social Doctrinal, 9, 15-32.

\section{LEGISLACIÓN Y PLANES DE ACCIÓN}

Convención de Naciones Unidas de Derechos de las Personas con Discapacidad, aprobada por la Asamblea de la ONU el 13 de diciembre de 2006, junto con su Protocolo Facultativo, ratificada por España mediante instrumento de ratificación publicado en el BOE de 21 de abril de 2008.

Orden SSI/1474/2014, de 28 de julio, por la que se modifica la Orden de 2 de noviembre de 2000, por la que se determina la composición, organización y funciones de los Equipos de Valoración y Orientación dependientes del Instituto de Mayores y Servicios Sociales y se desarrolla el procedimiento de actuación para la valoración del grado de discapacidad dentro del ámbito de la Administración General del Estado.

Real Decreto 1971/1999, de 23 de diciembre, de procedimiento para el reconocimiento, declaración y calificación del grado de discapacidad.

Real Decreto 1169/2003, de 12 de septiembre por el que se modifica el anexo I del R. D. 1971/1999 de 23 de diciembre, de procedimiento para el reconocimiento, declaración y calificación del grado de discapacidad.

Real Decreto 1856/2009, de 4 de diciembre, de procedimiento para el reconocimiento, declaración y calificación del grado de discapacidad, y por el que se modifica el Real Decreto 1971/1999, de 23 de diciembre.

Real Decreto Legislativo 1/2013, de 29 de noviembre, BOE de 3 de diciembre de 2013.

Orden 710/2000, de 8 de mayo, de la Consejería de Servicios Sociales, por la que se establece el procedimiento de actuación para la aplicación y desarrollo del Real Decreto 1971/1999, sobre Reconocimiento, Declaración y Calificación del Grado de Discapacidad.

Plan de Acción de la Comunidad de Madrid para las Personas con Discapacidad 2005-2008. Disponible en http://www.madrid.org/bvirtual/BVCM007014.pdf.

II Plan de Acción para las Personas con Discapacidad 2003-2007. Disponible en: http://sid.usal.es/idocs/F8/FDO6636/iipapcd2003_2007.pdf 